Homology, Homotopy and Applications, vol.10(2), 2008, pp.195-209

\title{
GRÖBNER BASES OF ORIENTED GRASSMANN MANIFOLDS
}

\author{
TOMOHIRO FUKAYA
}

\author{
(communicated by Gunnar Carlsson)
}

Abstract

For $n=2^{m+1}-4, m \geqslant 2$, we determine the cup-length of $H^{*}\left(\widetilde{G}_{n, 3} ; \mathbb{Z} / 2\right)$ by finding a Gröbner basis associated with a certain subring, where $\widetilde{G}_{n, 3}$ is the oriented Grassmann manifold $S O(n+3) / S O(n) \times S O(3)$. As an application, we provide not only a lower but also an upper bound for the LS-category of $\widetilde{G}_{n, 3}$. We also study the immersion problem of $\widetilde{G}_{n, 3}$.

\section{Introduction}

Let $R$ be a commutative ring. The cup-length of $R$ is defined to be the greatest number $n$ such that there exist $x_{1}, \ldots, x_{n} \in R \backslash R^{\times}$with $x_{1} \cdots x_{n} \neq 0$. We denote the cup-length of $R$ by $\operatorname{cup}(R)$. In particular, for a space $X$ and a commutative ring $A$, the cup-length of $X$ with coefficients in $A$ is defined to be $\operatorname{cup}\left(\tilde{H}^{*}(X ; A)\right)$. We denote it by $\operatorname{cup}_{A}(X)$. It is well-known that $\operatorname{cup}_{A}(X)$ is a lower bound for the LS-category of $X$.

The aim of this paper is to study $\operatorname{cup}_{\mathbb{Z} / 2}\left(\widetilde{G}_{n, 3}\right)$, where $\widetilde{G}_{n, k}$ is the oriented Grassmann manifold $S O(n+k) / S O(n) \times S O(k)$. Note that $\widetilde{G}_{n, k}$ is $(n k)$-dimensional. While the cohomology of $\widetilde{G}_{n, 2}$ is well-known, that of $\widetilde{G}_{n, 3}$ is vague. However, Korbaš [8] gave rough estimates for $\operatorname{cup}_{\mathbb{Z} / 2}\left(\widetilde{G}_{n, 3}\right)$ by considering the height of $w_{2} \in$ $H^{*}\left(\widetilde{G}_{n, 3} ; \mathbb{Z} / 2\right)$, where $w_{2}$ is the second Stiefel-Whitney class.

Remark 1.1. The notation of the oriented Grassmann manifold in this paper is different from that of [8]. Here $\widetilde{G}_{n, k}$ consists of oriented $k$-dimensional subspaces in Euclidean $(n+k)$-space $\mathbb{R}^{n+k}$.

The author studies $H^{*}\left(\widetilde{G}_{n, 3} ; \mathbb{Z} / 2\right)$ by considering Gröbner bases associated with a certain subring of $H^{*}\left(\widetilde{G}_{n, 3} ; \mathbb{Z} / 2\right)$. It seems that, in principle, the method of Gröbner bases works better in such complicated calculations than that of usual algebraic topology. The author employs a computer and carries a huge amount of calculations for finding the above Gröbner bases and then he dares to conjecture:

The author was supported by Grant-in-Aid for JSPS Fellows (19.3177) from Japan Society for the Promotion of Science.

Received April 25, 2007, revised August 1, 2008; published on October 24, 2008.

2000 Mathematics Subject Classification: Primary 55M30, Secondary 57T15, 13 P10.

Key words and phrases: cup-length, LS-category, Gröbner bases, immersion.

This article is available at http://intlpress.com/HHA/v10/n2/a10

Copyright (C) 2008, International Press. Permission to copy for private use granted. 


\section{Conjecture 1.2.}

$$
\operatorname{cup}_{\mathbb{Z} / 2}\left(\widetilde{G}_{n, 3}\right)=\left\{\begin{array}{lr}
2^{m+1}-3 & \text { when } 2^{m+1}-4 \leqslant n \leqslant 2^{m+1}+2^{m}-6, \\
2^{m+1}-3+k & \text { when } n=2^{m+1}+2^{m}-5+k, 0 \leqslant k \leqslant 2, \\
2^{m+1}+2^{m}+\cdots & \text { when } n=2^{m+1}+2^{m}+\cdots+2^{j}-2+k, \\
+2^{j+1}+2^{j-1}+k & 0 \leqslant k \leqslant 2^{j-1}-1 .
\end{array}\right.
$$

When $n=2^{m+1}-4(m \geqslant 2)$, our method works very well and we obtain:

Theorem 1.3. $\operatorname{cup}_{\mathbb{Z} / 2}\left(\widetilde{G}_{n, 3}\right)=n+1$ when $n=2^{m+1}-4(m \geqslant 2)$.

For dimensional reasons, we have

$$
\operatorname{cat}(X) \leqslant \frac{3}{2} n
$$

where $\operatorname{cat}(X)$ denotes the LS-category of a space $X$ normalized as $\operatorname{cat}(*)=0$. Theorem 1.3 gives not only lower bounds for cat $\left(\widetilde{G}_{n, 3}\right)$, but also refines the inequality (1). Actually we obtain:

Corollary 1.4. $n+1 \leqslant \operatorname{cat}\left(\widetilde{G}_{n, 3}\right)<\frac{3}{2} n$ when $n=2^{m+1}-4(m \geqslant 2)$. In particular, we have $\operatorname{cat}\left(\widetilde{G}_{4,3}\right)=5$.

We will give applications of Theorem 1.3 for the immersion problem of $\widetilde{G}_{n, 3}$. By the classical result of Whitney [12], we know that $\widetilde{G}_{n, 3}$ immerses into $\mathbb{R}^{6 n-1}$. We will show:

Theorem 1.5. The oriented Grassmann manifold $\widetilde{G}_{n, 3}$ immerses into $\mathbb{R}^{6 n-3}$ but not into $\mathbb{R}^{3 n+8}$ when $n=2^{m+1}-4(m \geqslant 3)$ and $\widetilde{G}_{4,3}$ immerses into $\mathbb{R}^{21}$ but not into $\mathbb{R}^{17}$.

Remark 1.6. Walgenbach [11] obtained better results on the non-immersion of $\widetilde{G}_{n, 3}$ : $\widetilde{G}_{n, 3}$ does not immerse into $\mathbb{R}^{4 n-2 m+3}$. On the other hand, due to R. Cohen [2], $\widetilde{G}_{n, 3}$ is known to be immersed into $\mathbb{R}^{6 n-m+1}$. Then Theorem 1.5 gives a better estimate when $m=2,3$.

The organization of this paper is as follows. In Section 2, we consider the double covering map $p_{n}: \widetilde{G}_{n, 3} \rightarrow G_{n, 3}$, where $G_{n, 3}$ is the unoriented Grassmann manifold $O(n+3) / O(n) \times O(3)$. We identify the subring $\operatorname{Im} p_{n}^{*}$ of $H^{*}\left(\widetilde{G}_{n, 3} ; \mathbb{Z} / 2\right)$ with a certain algebra $\mathbb{Z} / 2\left[\bar{w}_{2}, \bar{w}_{3}\right] / J_{n}$, where generators of $J_{n}$ are given. In Section 3 , setting $n=2^{m+1}-4(m \geqslant 2)$, we will give an explicit description of generators of the ideal $J_{n}$ by using the binary expansion. In Section 4, we compute a Gröbner basis of $J_{n}$ and obtain $\operatorname{cup}\left(\mathbf{I m} p_{n}^{*}\right)$. In Section 5 , we show that $\operatorname{cup}\left(\operatorname{Im} p_{n}^{*}\right)$ determines $\operatorname{cup}_{\mathbb{Z} / 2}\left(\widetilde{G}_{n, 3}\right)$ and compute it. As an application, we give an estimate for $\operatorname{cat}\left(\widetilde{G}_{n, 3}\right)$ and study the immersion problem of $\widetilde{G}_{n, 3}$.

\section{Acknowledgements}

We would like to thank Daisuke Kishimoto and Shizuo Kaji who read the manuscript carefully and gave us useful comments. In particular, it was Don Davis who pointed out an error in the argument of the immersion in our manuscript and gave us useful advice. 


\section{Cohomology of $\widetilde{G}_{n, 3}$}

We consider the double covering

$$
p_{n}: \widetilde{G}_{n, 3} \rightarrow G_{n, 3} .
$$

It will be shown that $\operatorname{cup}_{\mathbb{Z} / 2}\left(\widetilde{G}_{n, 3}\right)$ can be determined by $\operatorname{cup}\left(\operatorname{Im} p_{n}^{*}\right)$. Then we shall investigate $\operatorname{cup}\left(\operatorname{Im} p_{n}^{*}\right)$. The arguments in this section are based on the work of Korbaš [8].

The mod 2 cohomology of $B O(3)$ is given by

$$
H^{*}(B O(3) ; \mathbb{Z} / 2)=\mathbb{Z} / 2\left[w_{1}, w_{2}, w_{3}\right],
$$

where $w_{i}$ is the $i$-th universal Stiefel-Whitney class. Borel [1] showed that the canonical map $i: G_{n, 3} \rightarrow B O(3)$ induces an epimorphism

$$
i^{*}: H^{*}(B O(3) ; \mathbb{Z} / 2) \rightarrow H^{*}\left(G_{n, 3} ; \mathbb{Z} / 2\right) .
$$

Hereafter we denote $i^{*}\left(w_{i}\right)$ by the same symbol $w_{i}$ ambiguously.

One can easily see that the above double covering (2) induces the Wang sequence as:

$$
\cdots \longrightarrow H^{q-1}\left(G_{n, 3} ; \mathbb{Z} / 2\right) \stackrel{\cdot w_{1}}{\longrightarrow} H^{q}\left(G_{n, 3} ; \mathbb{Z} / 2\right) \stackrel{p_{n}^{*}}{\longrightarrow} H^{q}\left(\widetilde{G}_{n, 3} ; \mathbb{Z} / 2\right) \longrightarrow \cdots .
$$

Then we have

$$
\operatorname{Im} p_{n}^{*} \cong \mathbb{Z} / 2\left[w_{1}, w_{2}, w_{3}\right] /\left(w_{1}, \operatorname{Ker} i^{*}\right) .
$$

Let $\pi: \mathbb{Z} / 2\left[w_{1}, w_{2}, w_{3}\right] \rightarrow \mathbb{Z} / 2\left[w_{2}, w_{3}\right]$ be the abstract ring homomorphism defined by $\pi\left(w_{1}\right)=0, \pi\left(w_{2}\right)=w_{2}$ and $\pi\left(w_{3}\right)=w_{3}$. Then it induces the isomorphism

$$
\operatorname{Im} p_{n}^{*} \cong \mathbb{Z} / 2\left[\bar{w}_{2}, \bar{w}_{3}\right] / J_{n},
$$

where $\pi\left(\operatorname{Ker} i^{*}\right)=J_{n}$ and we denote $w_{i}$ in $H^{*}\left(\widetilde{G}_{n, 3} ; \mathbb{Z} / 2\right)$ by $\bar{w}_{i}$. Note that the commutative diagram

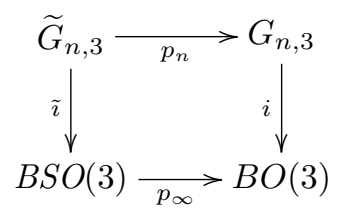

yields that $\tilde{\imath}^{*}\left(w_{i}\right)=\bar{w}_{i}$ for $i=2,3$ and $p_{\infty}^{*}: H^{*}(B O(3) ; \mathbb{Z} / 2) \rightarrow H^{*}(B S O(3) ; \mathbb{Z} / 2)$ is expressed by $\pi: \mathbb{Z} / 2\left[w_{1}, w_{2}, w_{3}\right] \rightarrow \mathbb{Z} / 2\left[w_{2}, w_{3}\right]$.

Let us give explicit generators of $J_{n}$. Borel [1] also showed that $\mathbf{K e r} i^{*}$ is generated by the homogeneous components of degrees $n+1, n+2$ and $n+3$ in

$$
\frac{1}{1+w_{1}+w_{2}+w_{3}} \text {. }
$$

Then it follows that $J_{n}$ is generated by the homogeneous components of degrees $n+1$, 
$n+2$ and $n+3$ in

$$
\frac{1}{1+\bar{w}_{2}+\bar{w}_{3}} \text {. }
$$

Let $N$ be the unique integer which satisfies $2^{N}<n \leqslant 2^{N+1}$. Since

$$
\operatorname{dim} \widetilde{G}_{n, 3}<4 n \leqslant 2^{N+3},
$$

we have

$$
\left(1+\bar{w}_{2}+\bar{w}_{3}\right)^{2^{N+3}}=1
$$

in $H^{*}\left(\widetilde{G}_{n, 3} ; \mathbb{Z} / 2\right)$. Then it follows that

$$
\frac{1}{1+\bar{w}_{2}+\bar{w}_{3}}=\left(1+\bar{w}_{2}+\bar{w}_{3}\right)^{2^{N+3}-1} ;
$$

hence $J_{n}$ is generated by

$$
g_{r}=\sum_{\frac{r}{3} \leqslant s \leqslant \frac{r}{2}}\left(\begin{array}{c}
s \\
3 s-r
\end{array}\right) \bar{w}_{2}^{3 s-r} \bar{w}_{3}^{r-2 s}
$$

for $r=n+1, n+2, n+3$.

\section{Investigating generators of $J_{n}$}

In this section, we investigate generators $g_{n+1}, g_{n+2}$ and $g_{n+3}$ of $J_{n}$ by exploiting the binary expansion.

Let us prepare notation for the binary expansion. To a non-negative integer $x$ with $0 \leqslant x<2^{k}$, we assign a sequence

$$
\epsilon_{k}(x)=\left(x_{k-1}, \ldots, x_{0}\right) \in\{0,1\}^{k}
$$

such that

$$
x=\sum_{i=0}^{k-1} x_{i} 2^{i}
$$

Equation (4) is, of course, the binary expansion of $x$. We denote $1-a$ by $\bar{a}$ with $a \in\{0,1\}$. For example, we have

$$
\epsilon_{k}\left(2^{k}-1\right)=(1, \ldots, 1)
$$

and

$$
\epsilon_{k}\left(2^{k}-1-x\right)=\left(\overline{x_{k-1}}, \ldots, \overline{x_{0}}\right)
$$

for $\epsilon(x)=\left(x_{k-1}, \ldots, x_{0}\right)$. We often denote $\left(x_{k}, \ldots, x_{0}\right) \in\{0,1\}^{k}$ by $\mathbf{x}_{k}$.

To calculate $\left(\begin{array}{c}s \\ 3 s-r\end{array}\right)$ modulo 2 , we use the following well-known result from elementary number theory.

Lemma 3.1. Let $n$ and $k$ be non-negative integers such that $k \leqslant n \leqslant 2^{l}-1$ and $\epsilon_{l}(n)=\left(n_{l-1}, \ldots, n_{0}\right), \epsilon_{l}(k)=\left(k_{l-1}, \ldots, k_{0}\right)$. Then we have $\left(\begin{array}{l}n \\ k\end{array}\right) \equiv 1(\bmod 2)$ if and only if $k_{i}=1$ implies $n_{i}=1$ for each $i$. 
In the rest of this paper, we assume that

$$
n=2^{m+1}-4(m \geqslant 2)
$$

Applying Lemma 3.1 to the coefficients of $g_{n+1}$, we have:

Proposition 3.2. $\left(\begin{array}{c}s \\ 3 s-(n+1)\end{array}\right)$ is even for all integers $s$ with $\frac{n+1}{3} \leqslant s \leqslant \frac{n+1}{2}$; that is, $g_{n+1}=0$.

Proof. Let $\epsilon_{m}(s)=\left(s_{m-1}, \ldots, s_{0}\right)$ for $\frac{n+1}{3} \leqslant s \leqslant \frac{n+1}{2}$ and let $\epsilon_{m+1}(n+1-2 s)=$ $\left(t_{m}, \ldots, t_{0}\right)$. Since $s \leqslant 2^{m}-2$, there exists an integer $i$ such that $s_{i}=0$. Let $i$ be the least integer satisfying $s_{i}=0$; that is, $\epsilon_{m}(s)=\left(s_{m-1}, \ldots, s_{i+1}, 0,1, \ldots, 1\right)$. Then it is easy to show that $t_{i}=1$. Hence it follows from Lemma 3.1 that

$$
\left(\begin{array}{c}
s \\
3 s-(n+1)
\end{array}\right)=\left(\begin{array}{c}
s \\
n+1-2 s
\end{array}\right) \equiv 0 \quad(\bmod 2) .
$$

Next we investigate $g_{n+2}$. Coefficients of $g_{n+2}$ are well understood by considering their binary expansion as in the above case of $g_{n+1}$. Let

$$
\begin{aligned}
\mathcal{S}_{k}=\left\{s \in \mathbb{Z} \mid \frac{n(k)+2}{3} \leqslant s \leqslant \frac{n(k)+2}{2} \text { and } \epsilon_{k}(s)=\left(s_{k-1}, \ldots, s_{0}\right)\right. \text { is such that } \\
\text { if } \left.s_{j}=0 \text {, then } s_{j+1}=1\right\} ;
\end{aligned}
$$

here $n(k)=2^{k+1}-4$. Note that $\frac{n(k)+2}{3} \leqslant s \leqslant \frac{n(k)+2}{2}$ implies that $s_{k-1}$ is always equal to 1 for each $s \in \mathcal{S}_{k}$ with $\epsilon_{k}(s)=\left(s_{k-1}, \ldots, s_{0}\right)$. There is a one-to-one correspondence between non-zero coefficients of $g_{n+2}$ and $\mathcal{S}_{m}$ as:

Lemma 3.3. $\left(\begin{array}{c}s \\ 3 s-(n+2)\end{array}\right) \equiv 1(\bmod 2)$ if and only if $s \in \mathcal{S}_{m}$.

Proof. Let $\epsilon_{m}(s)=\left(s_{m-1}, \ldots, s_{0}\right)$ for $\frac{n(m)+2}{3} \leqslant s \leqslant \frac{n(m)+2}{2}$. Then we have

$$
\epsilon_{m+1}(n+2-2 s)=\left(\overline{s_{m-1}}, \ldots, \overline{s_{0}}, 0\right) ;
$$

hence Lemma 3.3 follows from Lemma 3.1.

It is convenient for calculations in Section 4 to index coefficients of $g_{n+2}$ by exponents of $\bar{w}_{2}$ in (3); that is, $3 s-(n+2)$, not by $s \in \mathcal{S}_{m}$. Then we define a set $\mathcal{P}_{k}$ by

$$
\mathcal{P}_{k}=\left\{p \in \mathbb{Z} \mid p=3 s-(n(k)+2), s \in \mathcal{S}_{k}\right\} .
$$

$\mathcal{P}_{m}$ is expressed by the binary expansion as:

Proposition 3.4. Let

$$
\begin{array}{r}
\Delta_{k}=\left\{\left(p_{k-1}, \ldots, p_{0}\right) \in\{0,1\}^{k} \mid \text { if } p_{l-1}=1 \text { and } p_{l}=p_{l+1}=\right. \\
\quad \cdots=p_{l+2 t}=0 \\
\text { then } \left.p_{l+2 t+1}=0\right\} .
\end{array}
$$

Here we assume that $p_{-1}=1$. Then we have

$$
\mathcal{P}_{m}=\left\{p \in \mathbb{Z} \mid \epsilon_{m}(p) \in \Delta_{m}\right\} .
$$


We list some properties of $\Delta_{k}$ which will be useful in the following discussion. The proof is straightforward.

Proposition 3.5. The set $\Delta_{k}$ has the following properties:

(a) $\mathbf{p}_{k} \in \Delta_{k}$ implies $\left(1, \mathbf{p}_{k}\right) \in \Delta_{k+1}$.

(b) $\mathbf{p}_{k} \in \Delta_{k}$ implies $\left(\mathbf{p}_{k}, 1\right) \in \Delta_{k+1}$.

(c) $\Delta_{m}=\left\{\left(1, \mathbf{p}_{m-1}\right) \in\{0,1\}^{m} \mid \mathbf{p}_{m-1} \in \Delta_{m-1}\right\}$

$$
\sqcup\left\{\left(0,0, \mathbf{p}_{m-2}\right) \in\{0,1\}^{m} \mid \mathbf{p}_{m-2} \in \Delta_{m-2}\right\} .
$$

Proof of Proposition 3.4. Let $s \in \mathcal{S}_{m}$ with $\epsilon_{m}(s)=\left(s_{m-1}, \ldots, s_{0}\right)$. If $s_{m-2}=0$, then one has $s_{m-1}=1$ and $s_{m-3}=1$ by definition of $\mathcal{S}_{m}$. Then one can easily see that $s-2^{m-1} \in \mathcal{S}_{m-2}$ where $\epsilon_{m-1}\left(s-2^{m-1}\right)=\left(s_{m-2}, \ldots, s_{0}\right)$. If $s_{m-2}=1$, then one can see that $s-2^{m-1} \in \mathcal{S}_{m-1}$ as well. Hence one has obtained

$$
\mathcal{S}_{m}=\left\{s+2^{m-1} \mid s \in \mathcal{S}_{m-1}\right\} \sqcup\left\{s+2^{m-1} \mid s \in \mathcal{S}_{m-2}\right\} .
$$

We will show Proposition 3.4 by induction. We suppose that it is true for $m-1$ and $m-2$. Let $s \in \mathcal{S}_{m-1}$ and $p=3\left(s+2^{m-1}\right)-(n+2)$. By the hypothesis of the induction, $\epsilon_{m-1}\left(3 s-\left(n^{\prime}+2\right)\right)=\mathbf{p}_{m-1} \in \Delta_{m-1}$, where $n^{\prime}=2^{m}-4$. Since

$$
p=3\left(s+2^{m-1}\right)-(n+2)=3 s-2^{m}+2+2^{m-1}=3 s-\left(n^{\prime}+2\right)+2^{m-1},
$$

we have

$$
\epsilon_{m}(p)=\left(1, \mathbf{p}_{m-1}\right) \in \Delta_{m} .
$$

Similarly, let $s \in \mathcal{S}_{m-2}$ and $p=3\left(s+2^{m-1}\right)-(n+2)$. By the hypothesis of the induction, $\epsilon_{m-2}\left(3 s-\left(n^{\prime \prime}+2\right)\right)=\mathbf{p}_{m-2} \in \Delta_{m-2}$, where $n^{\prime \prime}=2^{m-1}-4$. Since

$$
p=3\left(s+2^{m-1}\right)-(n+2)=3 s-2^{m-1}+2=3 s-\left(n^{\prime \prime}+2\right),
$$

we have

$$
\epsilon_{m}(p)=\left(0,0, \mathbf{p}_{m-2}\right) \in \Delta_{m}
$$

Thus, by (5), we obtain

$$
\mathcal{P}_{m}=\left\{p+2^{m-1} \mid p \in \mathcal{P}_{m-1}\right\} \sqcup \mathcal{P}_{m-2}
$$

and, by (3.5) of Proposition 3.5, we have established Proposition 3.4.

For the rest of this section, we investigate $g_{n+3}$. Coefficients of $g_{n+3}$ can be well understood by using the binary expansion as well as above. Let

$$
\begin{array}{r}
\mathcal{S}_{k}^{\prime}=\left\{s^{\prime} \in \mathbb{Z} \mid \frac{n(k)+3}{3} \leqslant s^{\prime} \leqslant \frac{n(k)+3}{2} \text { and } \epsilon_{k}\left(s^{\prime}\right)=\left(s_{k-1}, \ldots, s_{1}, 1\right)\right. \text { is such that } \\
\text { if } \left.s_{j}=0, \text { then } s_{j+1}=1\right\} .
\end{array}
$$

Quite similarly to Lemma 3.3, we can see:

Lemma 3.6. $\left(\begin{array}{c}s^{\prime} \\ 3 s^{\prime}-(n+3)\end{array}\right) \equiv 1(\bmod 2)$ if and only if $s^{\prime} \in \mathcal{S}_{m}^{\prime}$. 
We give an explicit description of the set

$$
\mathcal{P}_{k}^{\prime}=\left\{p^{\prime} \in \mathbb{Z} \mid p^{\prime}=3 s^{\prime}-(n(k)+3), s^{\prime} \in \mathcal{S}_{k}^{\prime}\right\}
$$

as well. Define a map

$$
\iota: \mathcal{S}_{m-1} \rightarrow \mathcal{S}_{m}^{\prime}
$$

by $\iota(s)=2 s+1$. Then, obviously, it is bijective. Note that, for $s^{\prime}=\iota(s)$,

$$
3 s^{\prime}-(n+3)=3 \iota(s)-2^{m+1}+1=6 s-2^{m+1}+4=2\left(3 s-\left(n^{\prime}+2\right)\right),
$$

where $n^{\prime}=2^{m}-4$. Then we have $p \in \mathcal{P}_{m-1}$ if and only if $p^{\prime} \in \mathcal{P}_{m}^{\prime}$ such that $\epsilon_{m}\left(p^{\prime}\right)=\left(\mathbf{p}_{m-1}, 0\right)$ for $\epsilon_{m-1}(p)=\mathbf{p}_{m-1}$. Hence we have obtained:

Proposition 3.7. $\mathcal{P}_{m}^{\prime}=\left\{p \in \mathbb{Z} \mid \epsilon_{m}(p)=\left(\mathbf{p}_{m-1}, 0\right), \mathbf{p}_{m-1} \in \Delta_{m-1}\right\}$.

\section{Gröbner basis and cup-length}

In this section, by using the result of the previous section, we search for a Gröbner basis of $J_{n}$ in order to determine $\operatorname{cup}\left(\operatorname{Im} p_{n}^{*}\right)$.

\subsection{Gröbner bases}

We first recall the definition and some facts of Gröbner bases by restricting to our specific case. In order to clarify our discussion and to simplify notation, we shall make a convention of identifying a two variable polynomial ring with a certain set as follows. Let $\mathcal{X}=\left\{(p, q) \in \mathbb{Z}^{2} \mid p \geqslant 0, q \geqslant 0\right\}$ and let $P[\mathcal{X}]$ denote the set of finite subsets of $\mathcal{X}$. By assigning $F \in P[\mathcal{X}]$ to $\sum_{(p, q) \in F} \bar{w}_{2}^{p} \bar{w}_{3}^{q}$, we can identify $P[\mathcal{X}]$ with a polynomial ring $\mathbb{Z} / 2\left[\bar{w}_{2}, \bar{w}_{3}\right]$, and we shall make this identification throughout this section. This identification translates the operations in $\mathbb{Z} / 2\left[\bar{w}_{2}, \bar{w}_{3}\right]$ into $P[\mathcal{X}]$ as: For $F, G \in P[\mathcal{X}]$

$$
\begin{aligned}
F+G & =F \cup G \backslash F \cap G, \\
F \cdot G & =\sum_{(p, q) \in F,(r, s) \in G}(p+r, q+s) .
\end{aligned}
$$

This translation of operations enables us to handle the following polynomial calculations easily.

The order of $\mathcal{X}$ is given by the usual lexicographic order. Namely, for $(p, q),(r, s) \in \mathcal{X}$

$$
(p, q) \geqslant(r, s) \text { if and only if } p>r \text { or } p=r, q \geqslant s .
$$

By employing this order, we search for a Gröbner basis of the ideal $J_{n} \subset P[\mathcal{X}]$.

In order to define Gröbner bases, we prepare some notation and terminology. The leading term of a polynomial $F \in P[\mathcal{X}]$ is the monomial

$$
\operatorname{LT}(F)=\max \{(p, q) \in F\} .
$$

If there is a monomial $(p, q) \in \mathcal{X}$ such that $(p, q) \cdot \operatorname{LT}(G) \in F$, then the polynomial $F-(p, q) \cdot \operatorname{LT}(G)$ is called the remainder of $F$ on division by $G$. We denote the 
remainder $R=F-(p, q) \cdot \mathrm{LT}(G)$ of $F$ on division by $G$, by

$$
F \stackrel{G_{*}}{\longrightarrow} R .
$$

Choose $F_{1}, \ldots, F_{s} \in P[\mathcal{X}]$ and give them an arbitrary order. Then it is known that there is an algorithm to provide the decomposition of $F \in P[\mathcal{X}]$ as

$$
F=A_{1} F_{1}+\cdots+A_{s} F_{s}+R
$$

such that $A_{1}, \ldots, A_{s} \in P[\mathcal{X}]$ and $R$ is a linear combination of monomials, none of which is divisible by each $\operatorname{LT}\left(F_{1}\right), \ldots, \operatorname{LT}\left(F_{s}\right)$. The above $R$ is called the remainder of $F$ on division by $\left\{F_{1}, \ldots, F_{s}\right\}$ as well. However, this decomposition depends on the choice of an order of $F_{1}, \ldots, F_{s}$, and $F \in\left(F_{1}, \ldots, F_{s}\right)$ does not imply the remainder $R=0$. We can overcome this difficulty of remainders by choosing a Gröbner basis defined as:

Definition 4.1. Let $I$ be an ideal of $P[\mathcal{X}]$. A finite subset $G=\left\{G_{1}, \ldots, G_{s}\right\}$ is a Gröbner basis of $I$ if

$$
(\{\mathrm{LT}(F) \mid F \in I\})=\left(\operatorname{LT}\left(G_{1}\right), \ldots, \operatorname{LT}\left(G_{s}\right)\right) .
$$

Theorem 4.2. Let $I$ be an ideal of $P[\mathcal{X}]$ and let $\left\{G_{1}, \ldots, G_{s}\right\}$ be a Gröbner basis of $I$. Then the remainder of $F \in I$ on division by $\left\{G_{1}, \ldots, G_{s}\right\}$ is zero.

Buchberger [3] gave a criterion for a set of polynomials being a Gröbner basis of the ideal generated by it as follows. For $F, G \in P[\mathcal{X}]$, the least common multiple of $F$ and $G$ is the monomial

$$
\operatorname{LCM}(F, G)=(\max \{p, r\}, \max \{q, s\}),
$$

where $\operatorname{LT}(F)=(p, q)$ and $\operatorname{LT}(G)=(r, s)$. The $S$-polynomial of $F$ and $G \in P[\mathcal{X}]$ is

$$
S(F, G)=\frac{\operatorname{LCM}(F, G)}{\operatorname{LT}(F)} F+\frac{\operatorname{LCM}(F, G)}{\operatorname{LT}(G)} G .
$$

Theorem $4.3([3])$. The set of polynomials $\left\{G_{1}, \ldots, G_{s}\right\} \subset P[\mathcal{X}]$ is a Gröbner basis of the ideal $\left(G_{1}, \ldots, G_{s}\right)$ if and only if the remainder of $S\left(G_{i}, G_{j}\right)$ on division by $\left\{G_{1}, \ldots, G_{s}\right\}$ is zero for each $i \neq j$.

\subsection{Search for a Gröbner basis of $J_{n}$}

The author found the following polynomials experimentally by a computer calculation. For non-negative integers $i, t$ with $t-2\left(2^{m}-2^{i}\right) \equiv 0(\bmod 3)$, we define a polynomial $P(t, i)$ by

$$
\begin{aligned}
P(t, i) & =\left\{\left(p, \frac{t-2 p}{3}\right) \in \mathcal{X} \mid \epsilon_{m}(p)=(\mathbf{p}_{m-i}, \overbrace{0, \ldots, 0}^{i}), \mathbf{p}_{m-i} \in \Delta_{m-i}\right\}, \\
P_{i} & =P\left(2^{i}+n+1, i\right) .
\end{aligned}
$$

We shall prove that $\left\{P_{0}, \ldots, P_{m}\right\}$ is a Gröbner basis of $J_{n}$. 
In order to investigate $P_{i}$, we define the following sets which will be useful for expression. Let $\Delta(i, j, l)$ and $\bar{\Delta}(i, l)$ be

$$
\begin{aligned}
& \Delta(i, j, l)=\left\{(\mathbf{p}_{m-j}, \mathbf{p}_{j-i-l}, \overbrace{1, \ldots, 1}^{l}, \overbrace{0, \ldots, 0}^{i})\right. \in\{0,1\}^{m} \mid\left(\mathbf{p}_{m-j}, \mathbf{p}_{j-i-l}\right) \\
&\left.\in \Delta_{m-i-l}, \mathbf{p}_{j-i-l} \neq(1, \ldots, 1)\right\}, \\
& \bar{\Delta}(i, l)=\left\{(\mathbf{p}_{m-i-l-2}, 0,0, \overbrace{1, \ldots, 1}^{l}, \overbrace{0, \ldots, 0}^{i}) \in\{0,1\}^{m} \mid \mathbf{p}_{m-i-l-2} \in \Delta_{m-i-l}\right\} .
\end{aligned}
$$

It is easy to check:

Lemma 4.4. $\Delta(i, j, l)=\bar{\Delta}(i, l) \sqcup \Delta(i, j, l+1)$.

Let us begin investigating $P_{i}$. It is easy to verify that

$$
\operatorname{LT}\left(P_{i}\right)=\left(2^{m}-2^{i}, 2^{i}-1\right) \text {. }
$$

Proposition 4.5. We have $P_{0}, \ldots, P_{m} \in J_{n}$. In particular $P_{0}=g_{n+2}, P_{1}=g_{n+3}$.

Proof. By Propositions 3.4 and 3.7, one has $P_{0}=g_{n+2}$ and $P_{1}=g_{n+3}$. For $i<j$, it follows from (6) that

$$
\begin{aligned}
S\left(P_{i}, P_{j}\right)= & \left(0,2^{j}-2^{i}\right) \cdot P_{i}+\left(2^{j}-2^{i}, 0\right) \cdot P_{j} \\
= & \left\{\left(p, q_{i, j}\right) \in \mathcal{X} \mid \epsilon_{m}(p)=(\mathbf{p}_{m-j}, \mathbf{p}_{j-i}, \overbrace{0, \ldots, 0}^{i}),\left(\mathbf{p}_{m-j}, \mathbf{p}_{j-i}\right) \in \Delta_{m-i}\right\} \\
& +\left\{\left(p, q_{i, j}\right) \in \mathcal{X} \mid \epsilon_{m}(p)=(\mathbf{p}_{m-j}, \overbrace{1, \ldots, 1}^{j-i}, \overbrace{0, \ldots, 0}^{i}), \mathbf{p}_{m-j} \in \Delta_{m-j}\right\} \\
= & \left\{\left(p, q_{i, j}(p)\right) \in \mathcal{X} \mid \epsilon_{m}(p) \in \Delta(i, j, 0)\right\},
\end{aligned}
$$

where

$$
q_{i, j}(p)=\frac{3 \cdot 2^{j}-2 \cdot 2^{i}+n+1-2 p}{3} .
$$

By the definition of $\Delta_{k}$, one can easily see that $\Delta(i, 0, i+1)=\Delta_{m-i-2}$. Then it follows that $S\left(P_{i}, P_{i+1}\right)=P_{i+2}$; hence we have established Proposition 4.5.

We calculate the remainders of $S\left(P_{i}, P_{j}\right)$ on division by $\left\{P_{0}, \ldots, P_{m}\right\}$.

Lemma 4.6. The remainder of $Q_{i, j, l}=\left\{\left(p, q_{i, j}(p)\right) \in \mathcal{X} \mid \epsilon_{m}(p) \in \Delta(i, j, l)\right\}$ on division by $P_{i+l+2}$ is $Q_{i, j, l+1}$.

Proof. Let $p(i, l)$ be $\epsilon_{m}(p(i, l))=(\overbrace{1, \ldots, 1}^{m-i-l-2}, 0,0, \overbrace{1, \ldots, 1}^{l}, \overbrace{0, \ldots, 0}^{i})$. Then it is easy to 
see

$$
\operatorname{LT}(Q(i, j, l))=\left(p(i, l), q_{i, j}(p(i, l))\right)
$$

and it follows from (6) that

$$
\operatorname{LT}\left(P_{i+l+2}\right)=\left(p(i+l, 0), q_{i+j+2, i+j+2}(p(i+l, 0))\right) .
$$

Hence we have

$$
\left(2^{i+l}-2^{i}, 2^{j}-2^{i+l+1}\right) \cdot \operatorname{LT}\left(P_{i+l+2}\right)=Q(i, j, l) .
$$

On the other hand, one can easily check that

$$
\left(2^{i+l}-2^{i}, 2^{j}-2^{i+l+1}\right) \cdot P_{i+l+2}=\left\{\left(p, q_{i, j}(p)\right) \in \mathcal{X} \mid \epsilon_{m}(p) \in \bar{\Delta}(i, l)\right\}
$$

and then it follows from Lemma 4.4 that

$$
Q(i, j, l) \stackrel{P_{i+l+2 *}}{\longrightarrow} Q(i, j, l)+\left(2^{i+l}-2^{i}, 2^{j}-2^{i+l+1}\right) \cdot P_{i+l+2}=Q(i, j, l+1) .
$$

Theorem 4.7. The set $\left\{P_{0}, \ldots, P_{m}\right\}$ is a Gröbner basis of $J_{n}$.

Proof. By Proposition 4.5, we have $J_{n}=\left(P_{0}, \ldots, P_{m}\right)$. As in the proof of Proposition 4.5 , we have $S\left(P_{i}, P_{j}\right)=Q(i, j, 0)$, and then it follows from Lemma 4.6 that, for $i<j$,

$$
S\left(P_{i}, P_{j}\right)=Q(i, j, 0) \stackrel{P_{i+2_{*}}}{\longrightarrow} Q(i, j, 1) \stackrel{P_{i+3_{*}}}{\longrightarrow} \cdots \stackrel{P_{j_{*}}}{\longrightarrow} Q(i, j, j-i-1) \stackrel{P_{j+1_{*}}}{\longrightarrow} 0 .
$$

\subsection{Cup-length of $\operatorname{Im} p_{n}^{*}$}

In order to determine $\operatorname{cup}\left(\operatorname{Im} p_{n}^{*}\right)$, let us introduce new polynomials. For nonnegative integers $i, j, s$ with $s-2^{m+1}+2^{i+1} \equiv 0(\bmod 3)$, we define a polynomial $\hat{P}(s, i, j)$ by

$$
\hat{P}(s, i, j)=\left\{\left(p, \frac{s-2 p}{3}\right) \in \mathcal{X} \mid \epsilon(p) \in \bar{\Delta}(i, j)\right\} .
$$

Then we have

$$
\begin{aligned}
\mathrm{LT}\left(P_{i}\right)= & P\left(2^{i}+n+1, i\right)+P\left(2^{i}+n+1, i+2\right) \\
& +\sum_{1 \leqslant j \leqslant m-i-2} \hat{P}\left(2^{i}+n+1, i, j\right) .
\end{aligned}
$$

In order to investigate $\operatorname{cup}\left(\operatorname{Im} p_{n}^{*}\right)$, we shall calculate $\min \left\{p \mid(p, 0) \cdot \operatorname{LT}\left(P_{i}\right) \in J_{n}\right\}$ for each $i$ as follows.

Lemma 4.8. Let $\alpha_{i}=\min \left\{\alpha \mid(\alpha, 0) \cdot P(t, i) \in J_{n}\right\}$ for non-negative integers $i, t$ with

$$
2^{i-2}+n+1 \leqslant t<2^{i-1}+n+1, \quad t-2\left(2^{m}-2^{i}\right) \equiv 0 \quad(\bmod 3) .
$$

Then we have $\alpha_{i}=2^{m}-2^{i-1}$. In particular, $\alpha_{i}$ is independent from $t$ as above. 
Proof. Note that

$$
\begin{aligned}
& \left(2^{i-1}, 0\right) \cdot P(t, i) \\
& =\left\{\left(p, \frac{t+2^{i}-2 p}{3}\right) \in \mathcal{X} \mid \epsilon_{m}(p)=\left(\mathbf{p}_{m-i}, 1,0, \ldots, 0\right) \in\{0,1\}^{m},\left(\mathbf{p}_{m-i}, 1\right) \in \Delta_{m-i+1}\right\} \\
& \left(0, \frac{t+2^{i-1}-n-1}{3}\right) \cdot P_{i-1} \\
& =\left\{\left(p, \frac{t+2^{i}-2 p}{3}\right) \in \mathcal{X} \mid \epsilon_{m}(p)=\left(\mathbf{p}_{m-i+1}, 0, \ldots, 0\right) \in\{0,1\}^{m}, \mathbf{p}_{m-i+1} \in \Delta_{m-i+1}\right\} .
\end{aligned}
$$

and

By Proposition 3.5, we have

$$
\left(2^{i-1}, 0\right) \cdot P(t, i)+\left(0, \frac{t+2^{i-1}-n-1}{3}\right) \cdot P_{i-1}=P\left(t+2^{i}, i+1\right),
$$

and hence

$$
\left(2^{i-1}, 0\right) \cdot P(t, i) \stackrel{P_{i-1 *}}{\longrightarrow} P\left(t+2^{i}, i+1\right)
$$

Then we obtain

$$
\begin{gathered}
\left(2^{i-1}, 0\right) \cdot P(t, i) \stackrel{P_{i-1_{*}}}{\longrightarrow} P\left(t+2^{i}, i+1\right), \\
\left(2^{i}, 0\right) \cdot P\left(t+2^{i}, i+1\right) \stackrel{P_{i_{*}}}{\longrightarrow} P\left(t+2^{i}+2^{i+1}, i+2\right), \\
\vdots \\
\left(2^{m-1}, 0\right) \cdot P\left(t+2^{i}+\cdots+2^{m-1}, m\right) \stackrel{P_{m-1_{*}}}{\longrightarrow} 0 .
\end{gathered}
$$

We show that $\left(2^{m-1}-1,0\right) \cdot P\left(t+2^{i}+\cdots+2^{m-1}, m\right)$ does not lie in $J_{n}$. The following arguments are due to Korbaš. This polynomial corresponds to $\bar{w}_{2}^{2^{m-1}-1} \bar{w}_{3}^{\lambda}$ in $H^{*}\left(\widetilde{G}_{n, 3} ; \mathbb{Z} / 2\right)$ where $\lambda=\frac{1}{3}\left\{t+2^{m}-2^{i}\right\}$. Suppose $\bar{w}_{2}^{2^{m-1}-1} \bar{w}_{3}^{\lambda}=0$ in $H^{*}\left(\widetilde{G}_{n, 3} ; \mathbb{Z} / 2\right)$. Then $w_{2}^{2^{m-1}-1} w_{3}^{\lambda}$ lies in an ideal of $H^{*}\left(G_{n, 3} ; \mathbb{Z} / 2\right)$ generated by $w_{1}$ (see the exact sequence in Section 2). This contradicts the following facts on the multiplicative structure of $H^{*}\left(G_{n, 3} ; \mathbb{Z} / 2\right)$ by Stong [10]:

$$
\begin{aligned}
w_{1}^{2^{m+1}-1} w_{2}^{2^{m-1}-1} w_{3}^{n-2^{m}+1} & \neq 0 \\
w_{1}^{2^{m+1}} & =0 .
\end{aligned}
$$

This completes the proof of Lemma 4.8 .

Lemma 4.9. Let $\alpha_{i}$ be as in Lemma 4.8. Then we have $\left(\alpha_{i}, 0\right) \cdot \hat{P}(s, i, j) \in J_{n}$.

Proof. Quite similarly to the proof of Lemma 4.8, one has

$$
\left(2^{j+i}+2^{i}, 0\right) \cdot \hat{P}(s, i, j)+\left(0, \frac{s+2^{i+1}+1}{3}\right) \cdot P_{j+i+1}=P\left(s+2^{j+i+1}+2^{i+1}, j+i+3\right) .
$$

By Lemma 4.8 , we have $2^{j+i}+2^{i}+\alpha_{j+i+3}<\alpha_{i}$, and then Lemma 4.9 is accomplished.

It follows from Lemmas 4.8 and 4.9 that:

Proposition 4.10. Let $\alpha_{i}$ be as in Lemma 4.8. Then we have

$$
\alpha_{i+1}=\min \left\{\alpha \mid(\alpha, 0) \cdot \operatorname{LT}\left(P_{i}\right) \in J_{n}\right\} .
$$


Corollary 4.11. Let $\chi_{1}$ be a fixed integer such that

$$
2^{m+1}-2^{i+1}-2^{i+2} \leqslant \chi_{1}<2^{m+1}-2^{i}-2^{i+1}
$$

and let $\chi_{2}=\max \left\{z \mid\left(\chi_{1}, z\right) \notin J_{n}\right\}$. Then we have $\chi_{2}=2^{i+1}-2$.

Proof. Let $\left(p_{i}, q_{i}\right)=\operatorname{LT}\left(P_{i}\right)$. Then, by $(6)$ and Lemma 4.8, we have

$$
\begin{gathered}
\cdots>p_{i-1}+\alpha_{i+1}>p_{i}+\alpha_{i+2}>p_{i+1}+\alpha_{i+3} \cdots, \\
\cdots<q_{i-1}<q_{i}<q_{i+1}<\cdots .
\end{gathered}
$$

Hence, by Theorems 4.2 and 4.7, we have established that, for

$$
p_{i+1}+\alpha_{i+3}=2^{m+1}-2^{i+1}-2^{i+2} \leqslant \chi_{1}<2^{m+1}-2^{i}-2^{i+1}=p_{i}+\alpha_{i+2},
$$

one has $\chi_{2}=q_{i+1}-1=2^{i+1}-2$.

From Corollary 4.11, $\chi_{1}+\chi_{2}$ is maximized when $\left(\chi_{1}, \chi_{2}\right)=(n, 0)$, and the maximum value is $n$. The maximum value is equal to $\operatorname{cup}\left(\operatorname{Im} p_{n}^{*}\right)$. Therefore we have obtained:

Corollary 4.12. $\operatorname{cup}\left(\operatorname{Im} p_{n}^{*}\right)=n$. In particular, $\bar{w}_{2}^{n} \neq 0$.

\section{Cup-length of $\widetilde{G}_{n, 3}$ and its applications}

In this section, we determine $\operatorname{cup}_{\mathbb{Z} / 2}\left(\widetilde{G}_{n, 3}\right)$ and give its applications to immersion of $\widetilde{G}_{n, 3}$ into a Euclidean space.

Proof of Theorem 1.3. By Corollary 4.12, one has $\bar{w}_{2}^{n} \neq 0$. Then, by Poincaré duality, there exists $x \in H^{n}\left(\widetilde{G}_{n, 3} ; \mathbb{Z} / 2\right)$ such that $\bar{w}_{2}^{n} x \neq 0$, and hence we have $\operatorname{cup}_{\mathbb{Z} / 2}\left(\widetilde{G}_{n, 3}\right) \geqslant n+1$.

Note that the canonical map $\widetilde{G}_{n, 3} \rightarrow B S O(3)$ is an $n$-equivalence. Then it follows that $H^{*}\left(\widetilde{G}_{n, 3} ; \mathbb{Z} / 2\right) \cong \mathbf{I m} p_{n}^{*}$ in dimensions less than $n$. Now suppose that there exist $x_{1}, \ldots, x_{n+2} \in \widetilde{H}^{*}\left(\widetilde{G}_{n, 3} ; \mathbb{Z} / 2\right)$ such that $x_{1} \cdots x_{n+2} \neq 0$. By a dimensional reason, one has $\left|x_{i}\right|<n$ for each $i$, and then this contradicts to Corollary 4.12. Hence we have obtained Theorem 1.3.

Proof of Corollary. From Theorem 1.3 and the inequality $\operatorname{cup}_{\mathbb{Z} / 2}\left(\widetilde{G}_{n, 3}\right) \leqslant \operatorname{cat}\left(\widetilde{G}_{n, 3}\right)$, it follows that $n+1 \leqslant \operatorname{cat}\left(\widetilde{G}_{n, 3}\right)$.

Note that $\bar{w}_{2} \in H^{2}\left(\widetilde{G}_{n, 3} ; \mathbb{Z} / 2\right)$ is the fundamental class in the sense of James [7]. By Corollary 4.12, we have $\bar{w}_{2}^{n+1}=0$, and then it follows from Proposition 5.3 in [7] that $\operatorname{cat}\left(\widetilde{G}_{n, 3}\right)<\frac{3}{2} n$. Hence we have established the corollary.

Let us consider the immersion of $\widetilde{G}_{n, 3}$ into a Euclidean space as applications of Theorem 1.3. Of course, as mentioned in Section 1, we know, by the result of Whitney [12], that $\widetilde{G}_{n, 3}$ immerses into $\mathbb{R}^{6 n-1}$. We shall give a slightly better estimate. 
We denote the canonical vector bundle over $\widetilde{G}_{n, 3}$ by $\gamma$ and a stable normal bundle of $\widetilde{G}_{n, 3}$ by $\nu$. We abbreviate the classifying map $\widetilde{G}_{n, 3} \rightarrow B S O(\infty)$ of $\nu$ by the same symbol $\nu$. It is well-known that $T \widetilde{G}_{n, 3}=\gamma \otimes \gamma^{\perp}$; then we have

$$
\begin{aligned}
T \widetilde{G}_{n, 3} \oplus \gamma \otimes \gamma & =\gamma \otimes \gamma^{\perp} \oplus \gamma \otimes \gamma \\
& =\gamma \otimes\left(\gamma^{\perp} \oplus \gamma\right) \\
& =(n+3) \gamma .
\end{aligned}
$$

By Corollary 4.12, we have $\left(1+\bar{w}_{2}+\bar{w}_{3}\right)^{n+4}=1$. Using the formula for the StiefelWhitney class of a tensor product shows that $w(\gamma \otimes \gamma)=1+\bar{w}_{2}^{2}+\bar{w}_{3}^{3}$. Since $\nu \oplus T \widetilde{G}_{n, 3}$ is trivial, we have

$$
\begin{aligned}
w(\nu) & =\frac{w(\gamma \otimes \gamma)}{w((n+3) \gamma)} \\
& =\frac{1+\bar{w}_{2}^{2}+\bar{w}_{3}^{2}}{\left(1+\bar{w}_{2}+\bar{w}_{3}\right)^{n+3}} \\
& =\left(1+\bar{w}_{2}^{2}+\bar{w}_{3}^{2}\right)\left(1+\bar{w}_{2}+\bar{w}_{3}\right) \\
& =1+\bar{w}_{2}+\bar{w}_{3}+\bar{w}_{2}^{2}+\bar{w}_{2}^{3}+\bar{w}_{3}^{2}+\bar{w}_{2}^{2} \bar{w}_{3}+\bar{w}_{2} \bar{w}_{3}^{2}+\bar{w}_{3}^{3} .
\end{aligned}
$$

Then it immediately follows that $\widetilde{G}_{n, 3}$ does not immerse into $\mathbb{R}^{3 n+8}$ for $n=2^{m+1}-4 m \geqslant 3$ and $\widetilde{G}_{4,3}$ does not immerse into $\mathbb{R}^{17}$.

Now let us consider the modified Postnikov tower of the fibration

$$
B S O(3 n-3) \rightarrow B S O(\infty),
$$

following Gitler and Mahowald [4]. We obtain the modified Postnikov tower of $B S O(3 n-3) \rightarrow B S O(\infty)$ in dimensions less than or equal to $3 n$ as:

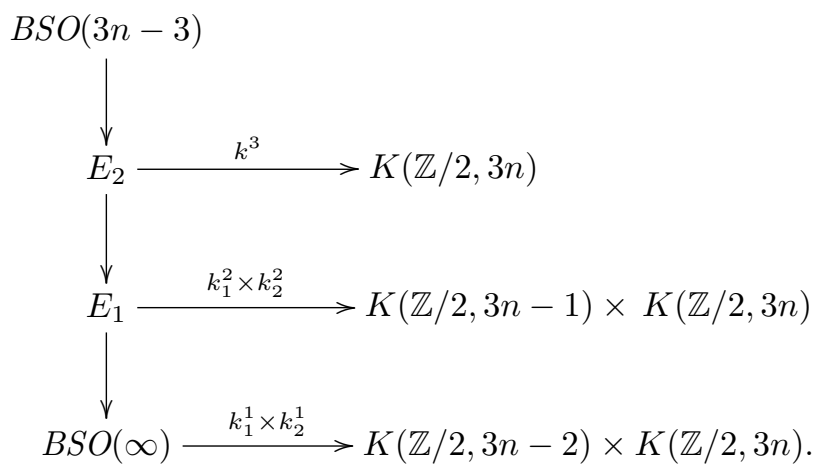

The $k$-invariants are as follows:

$$
\begin{aligned}
& k_{1}^{1}=w_{3 n-2}, \quad k_{2}^{1}=w_{3 n} \\
& k_{1}^{2}:\left(S q^{2}+w_{2}\right) k_{1}^{1}=0 \\
& k_{2}^{2}:\left(S q^{2} S q^{1}+w_{2} S q^{1}\right) k_{1}^{1}+S q^{1} k_{2}^{1}=0 \\
& k^{3}: S q^{1} k_{2}^{2}+\left(S q^{2}+w_{2}\right) k_{1}^{2}=0
\end{aligned}
$$

It is our goal to lift the map $\nu: \widetilde{G}_{n, 3} \rightarrow B S O(\infty)$ up to $B S O(3 n-3)$. 
It follows from (9) that $w_{3 n-2}(\nu)=w_{3 n}(\nu)=0$. Therefore the $k^{1}$-invariants of $\nu$ are zero, and so $\nu: \widetilde{G}_{n, 3} \rightarrow B S O(\infty)$ lifts up to $\nu^{\prime}: \widetilde{G}_{n, 3} \rightarrow E_{1}$. The $k^{2}$-invariants are $k_{1}^{2}(\nu) \in H^{3 n-1}\left(\widetilde{G}_{n, 3} ; \mathbb{Z} / 2\right), \quad k_{2}^{2}(\nu) \in H^{3 n}\left(\widetilde{G}_{n, 3} ; \mathbb{Z} / 2\right)$ and the $k^{3}$-invariant is $k^{3}(\nu) \in H^{3 n}\left(\widetilde{G}_{n, 3} ; \mathbb{Z} / 2\right)$. By Poincaré duality, one has $H^{3 n-1}\left(\widetilde{G}_{n, 3} ; \mathbb{Z} / 2\right)=0$, so $k_{1}^{2}(\nu)=0$. To prove that the map $\nu^{\prime}$ lifts to $B S O(3 n-3)$, it is enough to show that $k_{2}^{2}(\nu)$ and $k^{3}(\nu)$ are in the indeterminacy. This requires knowing the Steenrod operations $S q^{1}$ on $H^{3 n-3}\left(\widetilde{G}_{n, 3} ; \mathbb{Z} / 2\right)$ and $S q^{2}$ on $H^{3 n-2}\left(\widetilde{G}_{n, 3} ; \mathbb{Z} / 2\right)$. Let $x_{i}$ be a generator of $H^{i}\left(\widetilde{G}_{n, 3} ; \mathbb{Z} / 2\right) \cong \mathbb{Z} / 2$ for $i=3 n-3,3 n-2$.

Lemma 5.1. $S q^{1} x_{3 n-3}=x_{3 n-2}$ and $S q^{2} x_{3 n-2}=0$.

Proof. Since $\widetilde{G}_{n, 3}$ and $B S O(3)$ are $n$-equivalent, $H_{2}\left(\widetilde{G}_{n, 3} ; \mathbb{Z}\right) \cong H_{2}(B S O(3) ; \mathbb{Z}) \cong$ $\pi_{2}(B S O(3))=\mathbb{Z} / 2$. By Poincaré duality, one has $H^{3 n-2}\left(\widetilde{G}_{n, 3} ; \mathbb{Z}\right)=\mathbb{Z} / 2$, so the Bockstein homomorphism

$$
\beta: H^{3 n-3}\left(\widetilde{G}_{n, 3} ; \mathbb{Z} / 2\right) \rightarrow H^{3 n-2}\left(\widetilde{G}_{n, 3} ; \mathbb{Z} / 2\right)
$$

must be an isomorphism. Therefore one has $S q^{1} x_{3 n-3}=\beta x_{3 n-3}=x_{3 n-2}$.

Consider the inclusion $i$ : $\widetilde{G}_{n-1,2} \hookrightarrow \widetilde{G}_{n, 3}$. Let $x \in H^{n}\left(\widetilde{G}_{n, 3} ; \mathbb{Z} / 2\right)$ be as in the proof of Theorem 1.3. Then $x$ is indecomposable. By a result of Ishitoya [6], it follows that

$$
i^{*}\left(S q^{2} x\right)=S q^{2} i^{*}(x)=e t,
$$

where $e=i^{*}(x) \in H^{n}\left(\widetilde{G}_{n-1,2} ; \mathbb{Z} / 2\right)$ and $t=i^{*}\left(\bar{w}_{2}\right) \in H^{2}\left(\widetilde{G}_{n-1,2} ; \mathbb{Z} / 2\right)$. From the proof of Theorem 1.3 and the Wu formula, we have $S q^{2} x_{3 n-2}=S q^{2} \bar{w}_{2}^{n-1} x=0$.

By above lemma, the indeterminacy of $k_{2}^{2}$ is $H^{3 n}\left(\widetilde{G}_{n, 3} ; \mathbb{Z} / 2\right)$. Therefore, we may choose $\nu^{\prime}$ such that $\nu^{\prime}$ lifts to $\nu^{\prime \prime}: \widetilde{G}_{n, 3} \rightarrow E_{2}$. The indeterminacy of $k^{3}$ is also $H^{3 n}\left(\widetilde{G}_{n, 3} ; \mathbb{Z} / 2\right)$ and so an appropriate choice of $\nu^{\prime \prime}$ lifts to $\bar{\nu}: \widetilde{G}_{n, 3} \rightarrow B S O(3)$. Hence we can see from a result of Hirsch [5] that $\widetilde{G}_{n, 3}$ immerses into $\mathbb{R}^{6 n-3}$. Thus one obtains Theorem 1.5.

\section{References}

[1] A. Borel, La cohomologie mod 2 de certains espaces homogènes, Comment. Math. Helv. 27 (1953), 165-197.

[2] R.L. Cohen, The immersion conjecture for differentiable manifolds, Ann. of Math. (2) 122 (1985), no. 2, 237-328.

[3] D. Cox, J. Little, and D. O'Shea, Ideals, varieties, and algorithms. An introduction to computational algebraic geometry and commutative algebra, second edition, Undergraduate Texts in Mathematics, Springer-Verlag, New York, 1997.

[4] S. Gitler and M. Mahowald, The geometric dimension of real stable vector bundles, Bol. Soc. Mat. Mexicana (2) 11 (1966), 85-107.

[5] M.W. Hirsch, Immersions of manifolds, Trans. Amer. Math. Soc. 93 (1959), 242-276.

[6] Kiminao Ishitoya, Squaring operations in the Hermitian symmetric spaces, J. Math. Kyoto Univ. 32 (1992), no. 1, 235-244. 
[7] I.M. James, On category, in the sense of Lusternik-Schnirelmann, Topology 17 (1978), no. 4, 331-348.

[8] J. Korbaš, Bounds for the cup-length of Poincaré spaces and their applications, Topology Appl. 153 (2006), no. 15, 2976-2986.

[9] K.G. Monks, Groebner bases and the cohomology of Grassmann manifolds with application to immersion, Bol. Soc. Mat. Mexicana (3) 7 (2001), no. 1, 123-136.

[10] R.E. Stong, Cup products in Grassmannians, Topology Appl. 13 (1982), no. 1, 103-113.

[11] M. Walgenbach, Lower bounds for the immersion dimension of homogeneous spaces, Topology Appl. 112 (2001), no. 1, 71-86.

[12] H. Whitney, The self-intersections of a smooth n-manifold in 2n-space, Ann. of Math. (2) 45 (1944), no. 2, 220-246.

Tomohiro Fukaya tomo_xi@math.kyoto-u.ac.jp

Department of Mathematics, Kyoto University, Kyoto 606-8502, Japan 